\title{
ESTUDIO DE LA APLICACIÓN DE LOS METAMATERIALES EN EL DISEÑO DE ATENAS TEXTILES
}

\author{
Claudia Herrera ${ }^{1}$, María Restrepo ${ }^{2}$, Leidy Sánchez ${ }^{3 .}$ \\ 1 Tecnóloga de Telecomunicaciones, marcela230108@gmail.com, \\ 2 Tecnóloga de Telecomunicaciones, mariae1001@gmail.com, \\ 3 Tecnóloga de Telecomunicaciones, xiomsanch@gmail.com. \\ 123 Instituto Tecnológico Metropolitano
}

\begin{abstract}
RESUMEN
Este articulo presenta una revisión documental concerniente al diseño de antenas textiles a través del uso de metamateriales; mencionando también los tipos de antenas, sus técnicas de implementación, su elaboración, los materiales para su construcción y la fuente de trabajo o sustento de cada una de ellas.Dichas antenas están construidas por metamateriales los cuales funcionan como un conductor textil compuestos con otros materiales textiles que trabajan como un sustrato y son favorables al ser livianas, flexibles, fáciles de producir, prácticamente económicas y fácilmente aplicable en una prenda. Estos textiles (e-textiles), cuentan con componentes electrónicos e interconexiones tejidas en ellos, tienen flexibilidad física y tamaño reducido, que no se pueden conseguir en otros tipos de técnicas de fabricación electrónica existentes.
\end{abstract}

Palabras claves: antenas textiles, metamateriales, innovación y tecnología

Recibido: 08 de Octubre de 2018. Aceptado: 14 de Noviembre de 2019

Received: October 08, 2018. Accepted: November 14, 2019

\section{STUDY OF APPLICATION OF METAMATERIALS IN DESIGN OF TEXTILE ATHENS}

\begin{abstract}
This document presents a documentary review concerning the design of textile antennas through the use of metamaterials; also mentioning the types of antennas, their implementation techniques, their preparation, the materials for their construction and the source of work or sustenance of each of them.Said antennas are constructed by metamaterials which function as a textile conductor composed with other textile materials that work as a substrate and are favorable because they are light, flexible, easy to produce, practically economical and easily applicable in a garment. These textiles (e-textiles), have electronic components and interconnections woven into them, have physical flexibility and typical size that can't be achieved in other types of existing electronic manufacturing techniques.
\end{abstract}

Keywords: textile antennas, metamaterials, innovation and technology

Cómo citar este artículo: C. Herrera, M. Restrepo, L. Sanchez "Estudio de la aplicación de los metamateriales en el diseño de atenas textiles", Revista Politécnica, vol. 15, no.29 pp.108-127, 2019. DOI: 10.33571/rpolitec.v15n29a9 


\section{INTRODUCCIÓN}

Teletranspórtanos, Scotty' es una de las órdenes más famosas de la historia del cine. El capitán de la nave estelar Enterprise podía hablar con su tripulación con sólo tocar la insignia de su camisa. Hoy día es posible hacer alusión a esta escena, gracias a la investigación e implementación de antenas textiles, las cuales, semejándose a un parche adherido a la ropa, van a permitir un alto grado de comunicación entre las personas a través de los sistemas de satélite Iridium y GPS, el primero permite la comunicación bidireccional de datos y voz mientras que el segundo va a permitir la localización y ubicación de cada persona en un lugar determinado.

Estas antenas surgen de la necesidad de los usuarios a la adaptación de nuevas redes de comunicación, iniciando en las redes PAN y llegando a las nuevas redes WBAN. También se han logrado adaptar a nuevos protocolos para el intercambio de información mayoritariamente, incrementando la adaptación y el sin fin de aplicaciones para estos dispositivos.

En este artículo, se presenta un estudio de los conceptos fundamentales referentes a las antenas textiles, así como también las aplicaciones de estas en el mercado tecnológico, estándares principales, desafíos a los que se enfrenta la implementación de esta tecnología, las herramientas utilizadas para su creación y comercialización, así como también los materiales importantes que permitan el desarrollo de dicha tecnología en conjunto con los aportes que puedan surgir de esta revisión [1].

\section{LOS METAMATERIALES}

Los metamateriales se definen en sentido amplio como estructuras artificiales, homogéneas desde el punto de vista electromagnético, que exhiben propiedades físicas insólitas no presentes en la naturaleza [2]. Según el Instituto Europeo Virtual para Materiales Electromagnéticos Artificiales y Metamateriales son «una disposición artificial de elementos estructurales, diseñada para conseguir propiedades electromagnéticas ventajosas e inusuales».

Como resultado, sólo se pueden obtener artificialmente en laboratorios por medio de una serie de métodos de fabricación y se caracterizan por tener unas propiedades macroscópicas distintas a las de sus materiales constituyentes básicos, las cuales dependen de su estructura, normalmente ordenada y no de su composición [2].

En otra perspectiva, los metamateriales poseen capacidades extraordinarias para curvar las ondas electromagnéticas [3]. Mientras que todos los materiales encontrados en la naturaleza tienen un índice de refracción positivo (el índice de refracción es una medida de cuánto se desvían las ondas electromagnéticas al pasar de un medio a otro). Los metamateriales presentan un índice de refracción negativo, lo que implica que las ondas incidentes se desvíen hacia el otro lado de la perpendicular a la superficie «normal» que separa los medios [4].

Por este motivo, también se les denomina como materiales «zurdos». Esto se debe a que los metamateriales tienen simultáneamente una permitividad eléctrica y una permeabilidad magnética negativas, por lo que el índice de refracción, $n$, toma un valor negativo [5]. Para que un metamaterial se comporte como un material homogéneo con un índice de refracción negativo, es condición necesaria que la dimensión máxima de sus componentes sea menor que la longitud de onda incidente.

En caso contrario, no serían metamateriales sino "cristales fotónicos», con un índice de refracción positivo. Es decir, que el signo del índice de refracción dependerá de la relación entre la dimensión máxima de los componentes del metamaterial y la longitud de onda incidente [6].

En definitiva, la capacidad teórica de los metamateriales para controlar el camino de propagación de todo tipo de ondas (principalmente electromagnéticas, pero también acústicas, 
sísmicas, etc.) harán posibles aplicaciones que al día de hoy resultan difíciles de imaginar [7].

Prácticamente, los metamateriales se utilizan para la ampliación de diversas estructuras que son periódicas y que a su vez realizan una combinación de los medios físicos como son permisividad eléctrica y permeabilidad magnética.

\subsection{Metamateriales: propiedades y características}

Las propiedades electromagnéticas de todos los materiales existentes en la naturaleza pueden ser determinadas a partir de dos parámetros, la permeabilidad magnética y la permitividad eléctrica, los cuales nos permiten caracterizar la respuesta de cualquier material cuando este interactúa con una onda electromagnética [8].

En principio, no existe límite para el rango de valores que estos dos parámetros pueden tomar. Por tanto, es posible diseñar y construir a voluntad materiales con características de respuesta electromagnética específicas no encontradas en la naturaleza [9]. Estos materiales fabricados en el laboratorio reciben el nombre genérico de metamateriales, y entre ellos se encuentran los conocidos por LHM, así llamados porque los vectores de campo de las ondas electromagnéticas que viajan en su interior están relacionados por la regla de la mano izquierda.

En cuanto a las características distintivas de los LHM, ciertas bandas de frecuencia presentan índice de refracción negativo con modos propagativos posibles. Este fenómeno se presenta solamente si ambos parámetros $\mu \mathrm{y} \varepsilon$, dentro de dichas bandas de frecuencias son negativos simultáneamente.

Así, por ejemplo, los metamateriales son materiales artificiales con propiedades electromagnéticas inusuales, que no se dan en medios naturales conocidos y cuyas características proceden de la estructuras diseñadas y difieren de las de sus componentes; generalmente se trata de estructuras periódicas con dimensiones menores que la longitud de onda incidente, de modo que la estructura diseñada se comporte como un medio efectivo y pueda ser modelada por formas de permisividad, permeabilidad, índice de refracción, etc. El propósito de este trabajo es presentar los principios y fundamentos de estos metamateriales de manera que despierte el interés de lectores no especializados.

\subsection{Aplicaciones de los metamateriales}

Los metamateriales prometen no sólo mejorar las prestaciones de un gran número de dispositivos y sistemas en los campos de la electrónica y de la optrónica, sino introducir cambios verdaderamente revolucionarios en un gran número de sectores relacionados con dichas tecnologías [10].

De forma puntual, la aplicación de los metamateriales ha incrementado en las últimas décadas, su utilización en cristales ópticos, electromagnéticos y diseño de líneas de transmisión; en la tecnología, su empleo en referencia al aprovechamiento de la energía solar, tipos de Atenas, entre otras. Son algunos de los ejemplos de la aplicación de estos materiales en la actualidad.

\subsubsection{Antenas basadas en metamateriales}

Los metamateriales son materiales diseñados típicamente con estructuras nuevas o artificiales para producir propiedades electromagnéticas que son inusuales o difíciles de obtener en la naturaleza. Debido a su promesa de proporcionar permitividad, permeabilidad e índice de refracción, los metamateriales han atraído un amplio interés y han llevado a una posible utilización en muchas aplicaciones electromagnéticas desde el régimen de microondas hasta el óptico, especialmente para los dispositivos de ondas radiadas [11].

Este artículo presenta una revisión detallada sobre los esfuerzos de investigación más recientes asociados con esas pequeñas antenas basadas en metamateriales. Se señalan algunas dificultades prácticas o limitaciones para el desarrollo de antenas [12]. Se incluye una amplia variedad de ejemplos de 
antenas para facilitar la comprensión de los lectores en general.

En otras palabras, las propiedades de los metamateriales son propicias para la fabricación de Atenas de recepción de señales y su aplicación en microondas hasta el óptico, que son elementos esenciales en la fabricación de estos.

\subsubsection{Desarrollo de metamateriales fotónicos tridimensionales}

Los metamateriales fotónicos son estructuras hechas por el hombre compuestas por bloques de construcción de longitud de onda metalodielectrica micro o nanoestructurada a medida. Este concepto engañosamente simple pero potente permite la realización de muchas propiedades ópticas nuevas e inusuales, como magnetismo en frecuencias ópticas, índice de refracción negativo, gran índice de refracción positivo, reflejo cero a través de adaptación de impedancia, absorción perfecta, dicroísmo circular gigante y propiedades ópticas no lineales mejoradas [11].

En este punto, las posibles aplicaciones de los metamateriales incluyen sistemas de imagen de resolución ultra alta, óptica de polarización compacta y dispositivos de camuflaje. Esta revisión describe el progreso reciente en la fabricación de estructuras metamateriales tridimensionales y analiza algunos de los desafíos restantes.

\subsubsection{Diseño de un sensor de permitividad dieléctrica relativa de un medio empleando una antena de microcinta con estructuras metamateriales}

Muchos de los sensores de microondas diseñados para medir la permitividad dieléctrica de materiales, pueden ofrecer información acerca de propiedades como temperatura, humedad, concentración, composición, densidad y otras, tal como lo han mostrado algunas investigaciones [13].

Entre estas técnicas, la más utilizada para dichas mediciones es la que emplea resonadores, entre otras cosas, porque presentan un buen factor de calidad y porque son altamente sensibles a los cambios del medio que los rodea [13]. Otro de los métodos electromagnéticos explorados consiste en la implementación de antenas de microcinta, que brindan flexibilidad en su diseño y permiten incluso realizar la construcción de sensores con tamaño reducido.

Cabe destacar, Esto constituye una ventaja debido a que no se requieren mucho volumen de las muestras que se desean analizar, por lo que la técnica es favorable desde el punto de vista económico. Otra ventaja es su sencillez, lo que la hace fácil de usar.

Usualmente, cuando se ha empleado esta técnica se ha usado línea de microcinta como dispositivo sensor, en la que el material bajo prueba se coloca sobre la microcinta generando una atenuación en la potencia de la antena. Además, la técnica brinda la posibilidad de realizar mediciones espectrales cuando se usa un resonador de microcinta, donde el método de medición se basa en calcular el corrimiento del espectro al cambiar el medio que está en contacto con el sensor [14]..



Fig.1. Antena para censado: (a) modelamiento de la antena; (b) discretizado de la antena

\section{Metodología}

Para el estudio llevado a cabo en este trabajo se analizó la variación del ancho de la línea de transmisión grabada sobre el plano tierra de la antena con el fin de hallar las dimensiones optimas, específicamente cuando la antena se emplea como elemento sensor. 
En la siguiente fase del trabajo, se realizó la simulación modelando un cambio de permitividad en el medio que rodea la antena de microcinta. la antena discretizada por medio de un enmallado triangular, diseñado especialmente para la antena que cumple con el criterio de que cada elemento debe tener dimensiones de $\lambda / 5$ para obtener resultados validos en el proceso de simulación. Todas las simulaciones se realizaron por medio del método de elementos finitos vectorial y utilizando el software de simulaciones electromagnéticas HFSS de Ansoft.

Así mismo, En la Figura 2 se muestra un esquema 2D de las caras frontal y posterior de la antena con estructura de metamateriales. Se observa que un anillo resonador de cobre ha sido definido sobre un substrato dieléctrico, al que se ha hecho una abertura que posteriormente se encarga de que la estructura tenga un comportamiento metamaterial, y cuya frecuencia de resonancia depende de sus dimensiones. Adicionalmente, sobre este mismo plano (parte superior del substrato) se modela una línea de alimentación con ajuste de impedancia, para garantizar que el dispositivo tenga una impedancia característica de $50 \Omega$. En la parte posterior de la antena se graba una línea de cobre de grosor $\Delta$ y longitud $41 \mathrm{~mm}$, para evaluar el comportamiento del sensor al variar el grosor de la línea, y que simultáneamente cumple la función de ser el plano tierra de la antena.

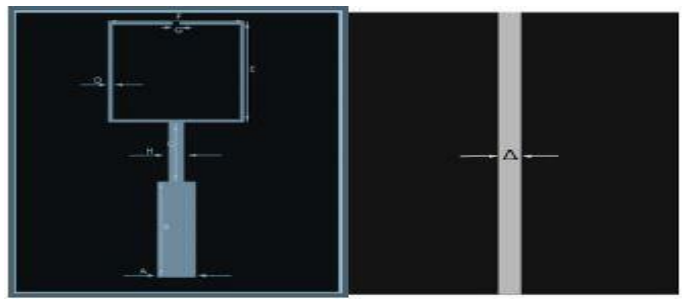

Fig.2. Esquema del sensor: vista frontal y vista posterior

\subsubsection{ATENAS TEXTILES}

\subsubsection{Materiales para la construcción de las antenas textiles}

Los materiales textiles generalmente tienen una muy baja constante dieléctrica, lo que reduce la superficie de las pérdidas de la onda y mejora el ancho de banda de la impedancia de la antena; por tanto, se requieren, básicamente, dos materiales: uno conductor y otro dieléctrico (o aislante), con los requisitos de que sean flexibles y moldeables para su posterior integración en las prendas de vestir [1].

De esta forma, se puede evidenciar que las propiedades electromagnéticas de estos metamateriales se encuentran en la naturaleza y éstas pueden ser identificadas a partir dos parámetros; su permeabilidad magnética y la permitividad eléctrica, las cuales hacen caracterizar las respuestas de cualquier material cuando este relacionándose con una onda electromagnética.

En particular, siendo el sustrato el material que se usa como una base para la fabricación del tag, el cual posee la antena y al microchip. Este componente, es la base fundamental del funcionamiento de la antena, ya que permite la formación de campos electromagnéticos alrededor de él (polarización del material) y desarrolla en la irradiación de la antena [16].

Una vez escogido el material a usar, se debe considerar la construcción de la antena donde los materiales deben ser unidos entre sí mediante un proceso en particular de acuerdo con el material seleccionado [17].

Cabe destacar, los metamateriales son usados para la construcción de antenas textiles, ya que normalmente poseen una muy baja constante dieléctrica, lo que reduce la superficie de las pérdidas de la onda y mejora el ancho de banda de la impedancia de la antena, gracias a la permeabilidad magnética y la permitividad eléctrica de estos materiales, en la actualidad existen varios tipos de antenas textiles.

\subsubsection{Tipos de antenas textiles}

Existe en el mercado variados tipos de antenas dentro de los cuales se puede mencionar 2 de ellos: 
Antenas de hilo: Son antenas donde los elementos radiantes son conductores de hilo. Se usan en las bandas MF, HF, VHF y UHF [18].

Antenas planas: Mayormente usadas para fabricar antenas textiles por su fácil integración en las prendas y porque se pueden emplear materiales textiles para su fabricación [19].

\subsubsection{Tipos de alimentación y sustento de las antenas textiles.}

\begin{tabular}{|c|c|c|}
\hline $\begin{array}{l}\text { Tipo de } \\
\text { alimentación }\end{array}$ & Ventajas & Desventajas \\
\hline $\begin{array}{l}\text { Cable coaxial } \\
\text { rígido }\end{array}$ & $\begin{array}{l}\text { Mayormente } \\
\text { usada porque } \\
\text { la punta del } \\
\text { coaxial se } \\
\text { podría colocar } \\
\text { en cualquier } \\
\text { punto de una } \\
\text { antena plana } \\
\text { para su } \\
\text { adaptación. }\end{array}$ & $\begin{array}{c}\text { No es flexible } \\
\text { y, por ende, no } \\
\text { se adapta a las } \\
\text { deformaciones } \\
\text { de la tela. }\end{array}$ \\
\hline & Conector co & \\
\hline $\begin{array}{c}\text { Línea de } \\
\text { alimentación } \\
\text { microstrip en } \\
\text { el substrato } \\
\text { de la antena } \\
\text { como } \\
\text { extensión del } \\
\text { propio } \\
\text { parche de la } \\
\text { antena }\end{array}$ & $\begin{array}{c}\text { Garantiza } \\
\text { estructura } \\
\text { plana (no } \\
\text { como el caso } \\
\text { del coaxial) y } \\
\text { permite el } \\
\text { montaje de } \\
\text { componentes } \\
\text { elec-trónicos } \\
\text { directamente } \\
\text { en la }\end{array}$ & $\begin{array}{l}\text { i) Posibles } \\
\text { radiaciones } \\
\text { parásitas de la } \\
\text { línea de } \\
\text { alimentación. } \\
\text { ii) Acoplamiento } \\
\text { electromagnético } \\
\text { parasitario entre } \\
\text { la antena, la } \\
\text { línea de }\end{array}$ \\
\hline
\end{tabular}






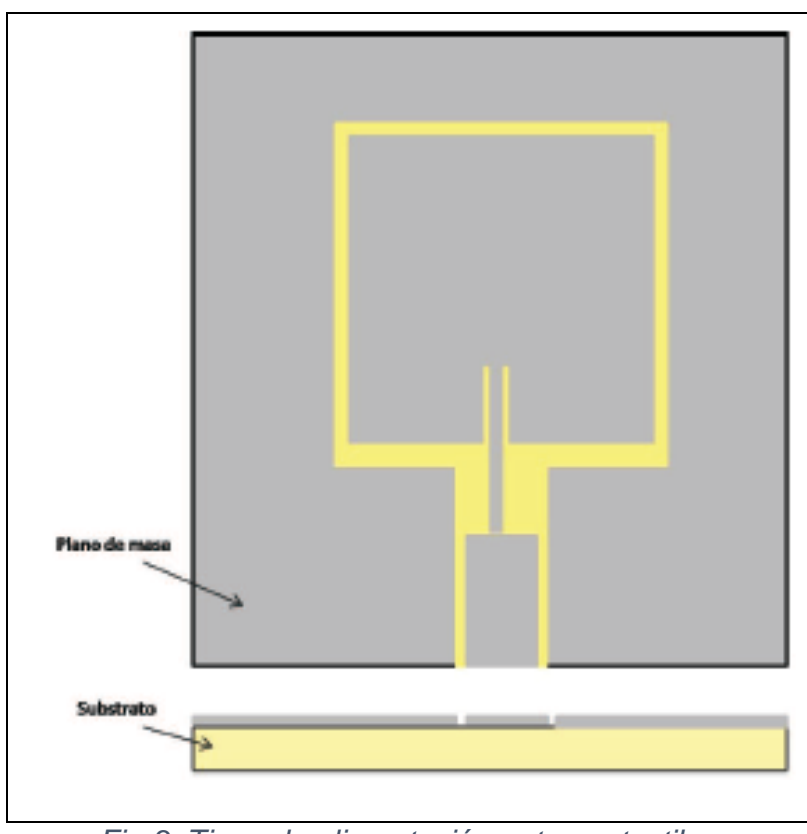

Fig.3. Tipos de alimentación antenas textiles

\subsubsection{Aplicaciones de los metamateriales y las propiedades textiles}

Los trabajos que se presentan a continuación, son una recopilación de las aplicaciones de las propiedades de los metamateriales en materiales textiles.

\subsection{Materiales textiles para el diseño de antenas portátiles}

En el amplio contexto de Wireless Body Sensor Networks para aplicaciones de salud y generalizadas, el diseño de antenas portátiles ofrece la posibilidad de monitoreo omnipresente, comunicación, recolección y almacenamiento de energía. Los requisitos específicos para las antenas portátiles son una estructura plana y materiales de construcción flexibles.

Ahora bien, varias propiedades de los materiales influyen en el comportamiento de la antena. Por ejemplo, el ancho de banda y la eficiencia de una antena de microbanda plana están determinados principalmente por la permitividad y el grosor del sustrato [20].
En definitiva, el uso de textiles en antenas portátiles requiere la caracterización de sus propiedades. Los textiles conductores eléctricos específicos están disponibles en el mercado y se han utilizado con éxito. Las telas textiles ordinarias se han utilizado como sustratos. Sin embargo, se puede encontrar poca información sobre las propiedades electromagnéticas de los textiles regulares. Por lo tanto, este trabajo se centra principalmente en el análisis de las propiedades dieléctricas de los tejidos normales [21].

En general, los textiles presentan una constante dieléctrica muy baja que reduce las pérdidas de onda de superficie y aumenta el ancho de banda de impedancia de la antena. Sin embargo, los materiales textiles intercambian constantemente moléculas de agua con el entorno, lo que afecta sus propiedades electromagnéticas. Además, las telas textiles son materiales porosos, anisotrópicos y compresibles cuyo espesor y densidad pueden cambiar a bajas presiones [22]. Por lo tanto, es importante saber cómo estas características influyen en el comportamiento de la antena para minimizar los efectos no deseados. Este documento presenta los puntos clave para el diseño y desarrollo de antenas textiles, desde la elección de los materiales textiles hasta el encuadre de la antena. También se presenta un análisis de los materiales textiles que se han utilizado.

En otras palabras, el uso de materiales textiles en antenas portátiles se basa en la especificación de cada una de sus propiedades, en general, los materiales textiles presentan una constante dieléctrica baja que reduce las pérdidas de onda de superficie y aumenta el ancho de banda, pero, los materiales textiles también comparten moléculas con regularidad, estas son moléculas de agua con el entorno, y esto incide en sus propiedades electromagnéticas.

\subsection{Antenas textiles Usable}

La investigación paramétrica estudia la influencia de una antena que se flexibiliza alrededor de su longitud y anchura en sus parámetros de rendimiento en la 
integración de cofres, piernas, brazos o muñecas para sitios inalámbricos de la red de área corporal (WBAN) [23].

A su vez, el gran desarrollo de dispositivos portátiles de bajo consumo ha dado el crecimiento de la necesidad de soluciones para la implementación de WBAN. Además de reducir las dimensiones del dispositivo y mejorar su consumo energético, también es importante abarcar el desarrollo de antenas flexibles que puedan integrarse en la ropa. Las antenas portátiles tienen un papel importante en el desarrollo de un WBAN, que presenta una amplia variedad de aplicaciones. Las antenas se pueden usar en sistemas que detectan el movimiento del cuerpo durante la actividad de ejercicio, monitoreando la frecuencia cardíaca y la presión sanguínea, y son aplicadas por un personal de respuesta de emergencia como bomberos o agentes de policía.

Las antenas son un elemento conductor textil integrado con otros materiales textiles que actúa como un sustrato y son favorables de ser livianas, flexibles, fáciles de producir, prácticamente económicas y fácilmente aplicable en una prenda [23].

En relación con lo anterior, los autores midieron la antena para varios radios de flexión, donde el rendimiento fue algo comprometido. Los valores de pérdida de retorno para simulaciones y mediciones en rangos extremos muestran que existe una desviación de $-5 \mathrm{~dB}$ en los resultados de medición. Se evaluaron la influencia del agua, lluvia y el agua de mar en el rendimiento de una antena en forma de ojo (parche cuadrado modificado) sobre sustrato de mezclilla para aplicaciones de banda ultra ancha.

Así, las aplicaciones de antenas amortiguadas e inmersas tuvieron un impacto importante en el rendimiento de la antena, por lo cual se propuso una que comprende una matriz $2 \times 2$ de elementos en forma de $L$ para el rango de frecuencia WBAN médica, donde se evalúa la influencia de flexión y se confirme que comprometería el rendimiento.

\section{Material de la antena}

El material usado para fabricar la antena es un tejido patentado de poliéster recubierto de cobre y níquel que está destinado principalmente a protegerlas contra la radiación de radiofrecuencia. Este es un tipo de material diseñado para antenas textiles ya que es flexible, liviano y fácil de cortar. Este material tiene una conductividad eléctrica que se calculó en función del espesor y la resistividad superficial, produciendo un valor de 28,570 S / m Denim fue el material de sustrato textil elegido para esta antena, ya que es más común, duradera, inelástica y de un bajo costo. Además, se puede integrar la antena textil desarrollada en la ropa, como en un bolsillo del pantalón [24].

En efecto, estas Atenas textiles están fabricadas con poliéster recubierto de cobre y níquel, esta es la combinación de los metamateriales y las telas. Se pueden usar en sistemas que detectan el movimiento y contienen también un sistema de monitoreo de frecuencias cardíaca y la presión sanguínea. Son bastante innovadoras además de fáciles de usar.

\subsection{Electrodos textiles en aplicaciones de detección de señal capacitiva}

Los ECG, EEG o EMG son señales que contienen información importante relacionada con el estado fisiológico de una persona y como tal, son usados en diversos métodos de diagnóstico [25]. La detección capacitiva de señales tiene varias ventajas, por ejemplo, no es necesario aplicar un gel electro conductor entre la piel y el electrodo, ya que reduce el tiempo de preparación para la medición, además este puede evitar irritación en la piel en caso de reacción alérgica al gel aplicado. Otros problemas influyentes son el secado del gel a lo largo del tiempo, especialmente en mediciones a largo plazo y el cortocircuito eléctrico entre los electrodos muy cercanos [26].

Con respecto a las propiedades y áreas de aplicación mencionadas, las mediciones capacitivas 
presentan métodos de adquisición de bioseñales cómodo, de acuerdo para mediciones a largo plazo con la capacidad de detectar señales a través de la ropa (ECG) y son adecuados para el diseño de sistemas de monitoreo portátiles.

En conjunto, el área del electrodo activo representa una placa de un condensador mientras que la superficie de la piel actúa como la segunda placa placa virtual del condensador [27]. El material dieléctrico, representado por la ropa, se coloca entre estas placas. La ropa está hecha principalmente de algodón o mezcla de algodón y poliéster. Hay algunos otros materiales que se pueden usar como la capa dieléctrica, $p$. capa dieléctrica de cerámica extraída del condensador o del cabello en el caso de medición de EEG [28].

Una comparación de 6 diferentes tipos de materiales textiles comunes son (algodón, lino, rayón, nylon, poliéster y PVC-textil), empleados como materiales dieléctricos en mediciones capacitivas.

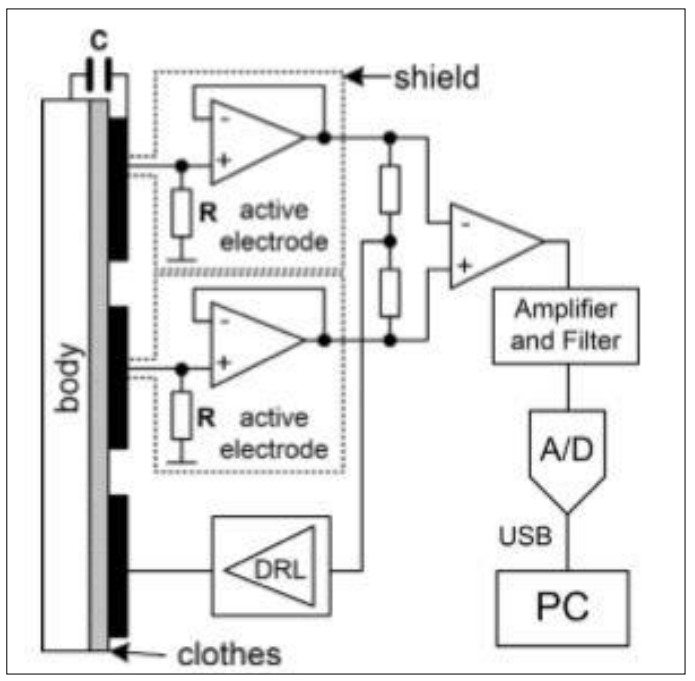

Fig.4. Diagrama de bloques del sistema de medición de bioseñales capacitiva

Vale la pena señalar, otras investigaciones aplican electrodos rígidos de placa metálica para la obtención de señales capacitivas, pero usar electrodos rígidos es incómodo, cuando se realiza monitoreo a largo plazo. En este aspecto, los electrodos textiles conductores presentan una alternativa más cómoda y flexible, incluida la adaptabilidad a los contornos del cuerpo.

\subsection{Antena de parche textil de doble banda para bandas GSM-WiFi usando EVA-Foam como sustrato}

Uno de los objetivos de los dispositivos portátiles es la integración en materiales flexibles como sustratos [29]. Bajo esta declaración, varios estudios han desarrollado la integración de elementos radiantes usando diferentes tipos de tejidos como sustrato y usando hilos conductores, o tejidos conducto como elemento radiante.

En la investigación de otros materiales que pueden usarse como sustratos en dispositivos portátiles, la espuma comercial de acetato de vinilo (EVA) aparece como un buen candidato debido a sus características físicas [30].

La espuma EVA es un polímero elastomérico que produce materiales muy similares al caucho en términos de suavidad y flexibilidad. Las características del EVA dependen del porcentaje de acetato de vinilo, que generalmente va del $10 \%$ al $40 \%$. El objetivo es conectar dispositivos portátiles a la red, la elección de las bandas debe tomarse para cubrir bandas comunes para la conexión de red, como las bandas GSM y WiFi.

\section{Caracterización eva-espuma}

Las investigaciones previas sobre la caracterización de la permitividad de la espuma EVA se hacen en frecuencias de banda $X$, en un primer enfoque, es importante caracterizar la espuma EVA como un sustrato para frecuencias inferiores a $3 \mathrm{GHz}$, donde se consiguen las bandas GSM y WiFi. Para la caracterización de la permitividad, se implementó un método que consiste en el uso de un resonador de cortocircuito abierto de micro banda de cuarto de longitud de onda para estimar la constante dieléctrica eficaz oeff del sustrato, en función de la frecuencia central y el ancho de banda del resonador (Qfactor). Para eso, se usó la siguiente ecuación [25]. 


$$
\epsilon_{\text {eff }}=\left(\frac{n \cdot c}{4 \cdot f_{0} \cdot L}\right)^{2}, n=1,3,5, \ldots
$$

Donde c es la velocidad de la luz en el espacio libre, $\mathrm{L}$ es la longitud del resonador de cuarto de onda y f0 es la frecuencia central del resonador definida por (fl + fh) / 2, donde fl es la frecuencia más baja de la 3Pérdida de inserción de $\mathrm{dB}$ y fh es la frecuencia superior de la pérdida de inserción de $3 \mathrm{~dB}$. Una vez obtenida la constante dieléctrica efectiva, la permitividad relativa oqr se obtiene usando la siguiente ecuación:

$$
\epsilon_{e f f}=\frac{\epsilon_{r}+1}{2}+\frac{\epsilon_{r}-1}{2}\left(1+12 \frac{h_{\text {sub }}}{W_{\text {line }}}\right)^{1 / 2}
$$

Resultados de diseño de antena y simulación

El diseño se trata de ranuras radiales que están situadas cerca del parche que irradia los bordes. Los modos son tm100 y tm300, ambos con patrones de radiación parecida. Donde $\mathrm{g}$ es un factor de corrección que desarrolla el efecto de carga de ranura. Finalmente, todas las dimensiones se mejoran utilizando el software ansoft hfss, para que sean adecuadas para la precisión de las herramientas de construcción disponibles en el laboratorio. Las dimensiones finales de la antena son las siguientes:

Wsub = Isub = $20 \mathrm{~cm} ;$ wpatch $=6,54 \mathrm{~cm}$; Ipatch $=6.21 \mathrm{~cm} ; \mathrm{w}=\mathrm{d}=0.05 \mathrm{~cm}$;

$\mathrm{I}=6.04 \mathrm{~cm}$;

feed $=1.35 \mathrm{~cm}$.

Para el diseño, se utilizaron los mismos valores de permitividad de espuma eva que para la simulación en la sección anterior (la variación lineal de la permitividad respeta la frecuencia de oq $=1,1 \mathrm{a}$ or $=$ 1,3 en la banda de 0,1 a 2,5 ghz). Finalmente, la altura del sustrato utilizada fue hsub $=0,172 \mathrm{~cm}$, que es la altura de la lámina comercial de eva. Con las dimensiones finales obtenidas, podemos simular los resultados del coeficiente de re flexión | s11 | y los patrones de radiación para ambas bandas [26].

Con las dimensiones finales obtenidas, podemos simular los resultados del coeficiente de re flexión | S11 | y los patrones de radiación para ambas bandas. La Figura 5 muestra el simulado.

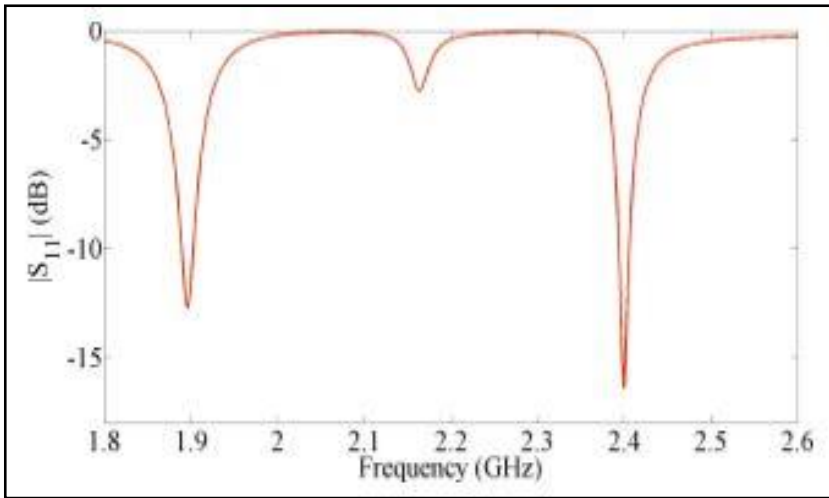

Fig.3. Simulado / S11 / parámetro de la antena parche textil de doble banda.

\section{Resultados de medición}

Los resultados de medición de la antena también se construyeron usando EVA foam como sustrato y Shieldex RNoraDell como el conductor del elemento radiante y el plano de tierra. Dejando las mismas dimensiones de la antena utilizada para la simulación

(Wsub $=$ Lsub $=20 \mathrm{~cm}$;

Wpatch $=6,54 \mathrm{~cm}$;

Lpatch $=6.21 \mathrm{~cm} ; \mathrm{w}=\mathrm{d}=0.05 \mathrm{~cm}$;

$\mathrm{I}=6.04 \mathrm{~cm}$;

feed $=1.35 \mathrm{~cm}$ ).

La alimentación de la antena se realizó con un conector SMA de $50 \Omega$. En la Figura 6 se observa la antena construida. El primer parámetro usado para medir es | S11 | de la antena de parche de doble banda textil. Ese realizó con un Vector Network Analyzer en una banda de frecuencia entre $1,8 \mathrm{GHz}$ y 2,5 GHz. Figura 6 Se observa el | S11 medido y 
simulado | como función de la frecuencia de la antena de parche.

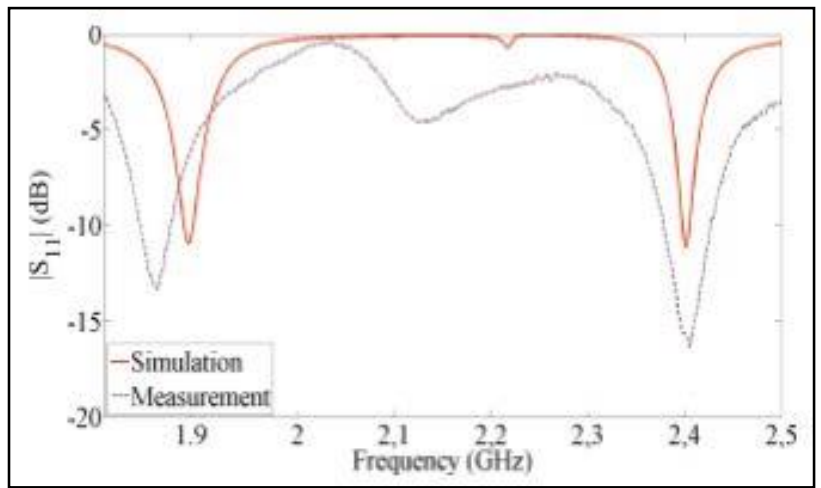

Fig.6. Simulado y medido / S11 / parámetro de la antena parche textil de doble banda

\subsection{Etiqueta de RFID UHF pasible de textiles basada en una antena de parche ranurado con interconexiones de tierra cosida y microchip}

La comunicación inalámbrica de datos digitales es una manera única de implementar sensores de antena y plataformas encendidas remotamente $y$ programables, con un microsistema de potencia de ultra baja integrado con una antena, proporciona funciones de identificación y detección en la tecnología actual [31]. Las etiquetas UHFRFID están abiertas de manera remota a varios metros de distancia más allá de los 20 metros en aplicaciones especializadas, ofreciendo un gran potencial para la identificación, la detección y el monitoreo. InWBANs, la tecnología de UHFRFID pasiva es previsible para facilitar el monitoreo a distancia de los parámetros de movimiento y fisiológicos, también para las aplicaciones de vida profunda, de camas bajas en hospitales y hogares de enfermo [32].

Cabe agregar, la materia biológica dieléctrica posee una conductividad eléctrica y una polarizabilidad notable. Llevando a la baja del rendimiento de radiación de las antenas mediante el consumo de energía en la interacción entre el campo electromagnético (EM) y el cuerpo, limitando grandemente la eficiencia de radiación alcanzable de antenas especiales de una sola capa [33].
En comparación, las estructuras de varias capas, como el parche de micro banda, las antenas $F$ invertidas (planar) y las antenas basadas en guías de onda integradas en sustrato y materiales metaméricos tienen beneficios del aislamiento de cuerpo incorporado en el plano del piso.

En este sentido, esta tecnología sería aceptable para la comodidad y las razones estéticas, pero también para la monitorización y consulta del paciente ejemplo, el cuidado de la demencia, donde los pacientes pueden tratar de eliminar dispositivos desconocidos. Sin embargo, otro parámetro importante radica en la fabricación de las interconexiones requeridas por un método que es compatible con el procesamiento de texto regular [34].

Fabricando una etiqueta RFID UHF pasiva e-textile aplicada en una antena de parcheo ranurada donde se crearon interconexiones de parche al suelo y de la antena a microchip con una máquina de coser, Metal e hilo plateado.

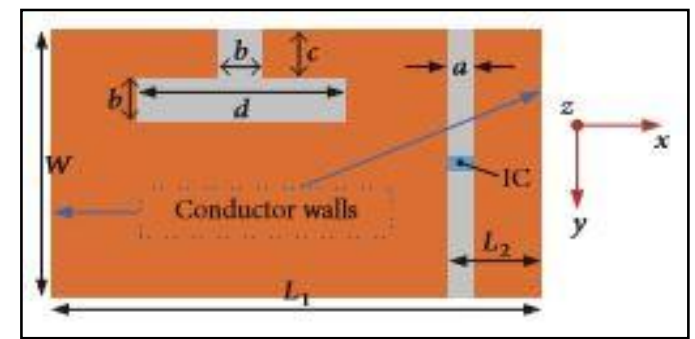

Fig.7. Diagrama estructural de la antena de parche ranurada. Dimensiones de la antena $(\mathrm{mm})$

Es necesario aclarar que, la implementación de etiquetas para producir comunicación inalámbrica de datos digitales es una manera única de implementar sensores de antena y plataformas encendidas remotamente y programables.

\subsection{Diseño de una antena multimodo sobre substrato textil para aplicaciones corporales}

El diseño de una antena sobre substrato textil que presente comportamiento multimodo operando a 2.45 GHz bajo el estándar Zigbee. La antena proporcionará comportamiento MIMO gracias a la 
excitación simultánea de varios modos de radiación con diagramas ortogonales, y será adecuada para aplicaciones corporales [35]. El substrato textil empleado será un tejido $100 \%$ algodón de características electromagnéticas desconocidas inicialmente. De aquí deriva otro de los objetivos principales del presente proyecto, que es la caracterización del textil empleado como substrato de la antena. Con el fin de determinar las propiedades dieléctricas del material textil se estudiarán distintas técnicas de caracterización.

El empleo de un substrato dieléctrico textil dotará a la antena de la flexibilidad necesaria para ser empleada en redes de sensores que trabajen en entornos de área corporal [35]. En base a la segunda fase del presente proyecto, se ha desarrollado un kit de medida con el objetivo de extraer las propiedades dieléctricas del material textil empleado como substrato dieléctrico de la antena. El kit está formado por un conjunto de líneas TFMSL (Thin Film Microstrip Lines) y su función es realizar la calibración del analizador de redes vectorial disponible en el Grupo de Radiaciones Electromagnéticas del iTEAM, así como la medida de los parámetros.

Considerando que, el objetivo del empleo de un substrato dieléctrico textil aportara a la antena flexibilidad, es necesario que tenga esta propiedad para poder ser empleada en redes de sensores que trabajen en entornos de área corporal. En este sentido, las propiedades textiles se emplean como substrato de la antena para determinar los componentes dieléctricos del material textil y así proceder a su utilización.

\subsection{Electrónica usable y textiles inteligentes: una crítica}

Los Textiles Electrónicos (e-textiles) son telas que cuentan con componentes electrónicos e interconexiones tejidas en ellos, que presentan flexibilidad física y tamaño típico que no se pueden lograr con otras técnicas de fabricación electrónica existentes [36].
Ya que visión detrás de la informática portátil prevé que los sistemas electrónicos futuros sean una parte integral de nuestros equipos cotidianos, dichos dispositivos electrónicos deben cumplir requisitos especiales relacionados con la usabilidad. Los sistemas portátiles se caracterizarán por su capacidad de reconocer automáticamente la actividad y el estado de comportamiento de su propio usuario, así como de la situación que lo rodea, y utilizar esta información para ajustar la configuración y la funcionalidad del sistema [37].

Nos centramos, en los avances recientes en el campo de Smart Textiles y presta especial atención a los materiales y su proceso de fabricación. Cada técnica muestra ventajas y desventajas y nuestro objetivo es destacar una posible compensación entre la flexibilidad, la ergonomía, el bajo consumo de energía, la integración y finalmente la autonomía.

En contraste con lo anterior, Los componentes y las interconexiones son intrínsecos al tejido y, por lo tanto, son menos visibles y no pueden enredarse ni engancharse con los objetos circundantes. Los textiles electrónicos también pueden adaptarse más fácilmente a los cambios rápidos en los requisitos computacionales y de detección de cualquier aplicación específica, lo que representa una característica útil para la administración de energía y la conciencia del contexto.

\subsection{Compacto Dual-Band Textile PIFA para bandas ISM de $433 \mathrm{MHz} / 2.4 \mathrm{GHz}$}

En el mundo se han investigados muchas topologías de antena, siendo las antenas textiles conformables FLEXIBLES las que han abarcado una gran atención en relación con el desarrollo de sistemas portátiles. Se han estudiado muchas topologías de antena, incluidos parches de micro banda, antenas planas invertidas-F (PIFA), antenas de guía de onda integradas de sustrato $y$ antenas basado en metamateriales [38].

Recientemente, varios investigadores han estudiado la relación entre el tamaño de la tierra y el ancho de las bandas. y han propuesto un diagrama de flujo de diseño basado en la teoría del modo característico. 
Este es un método de diseño que ya ha sido ampliamente utilizado en las llamadas "antenas de chasis" para terminales móviles [39].

Inspirándose en este concepto de diseño, se presenta una antena textil dirigida tanto a las bandas de $433 \mathrm{MHz}$ como a 2,4 GHzISM [40].

\section{Topología de antena y mecanismo operativo}

La topología de la antena propuesta, trata de introducir varias ranuras en el suelo para agrandar el ancho de las bandas. Introduciendo una ranura en forma de $L$ en el parche para lograr el funcionamiento en la banda de $2,4 \mathrm{GHz}$, Como sustrato se usa una capa de fieltro de $6 \mathrm{~mm}$ de espesor con una permitividad relativa de 1.3 y la tangente de pérdida de 0.044 . La capa metálica está diseñada con una lámina de conductividad de conductos de $0,17 \mathrm{~mm}$ de grosor con una conductividad de 1,18 × $105 \mathrm{~S} /$ capa superior de adhesivo secundario en la parte posterior [41].

Así mismo, su característica clave es aumentar el ancho de la banda alrededor de $433 \mathrm{MHz}$ al excitar el plano de tierra. Sin embargo, para permitir planos de tierra mucho más pequeños que la mitad de una longitud de onda a $433 \mathrm{MHz}$, las ranuras direccionales en y, se cortan en el suelo, lo que extiende la ruta de la corriente. Esto da como resultado una longitud de tierra de solo $140 \mathrm{~mm}$. Cabe destacar que el suelo se puede miniaturizar más cortando más ranuras, pero a costa de disminuir el ancho de banda correspondiente [42].

\section{Rendimiento en espacio libre}

Un PIFA muy pocas veces a traviesa tales bandas teniendo una topología compacta. El ancho de banda más amplio es de $308 \mathrm{MHz}$ (simulado) y $309 \mathrm{MHz}$ (medido), que es similar al de la banda ISM (de2.4 a $2.5 \mathrm{GHz}$ ). Una topología sin ranuras en el suelo también es similar y se comparó. Como resultado, la coincidencia es mucho más mala en la banda inferior, en la banda superior, casi no se ve afectada por las ranuras de tierra [43].

\subsection{Diseño de una microbanda de antena tri-banda para un WBAN de bajo SAR}

Este artículo presenta un nuevo diseño de una antena Tri-Band de microbanda para una baja SAR (tasa de absorción específica) de WBAN (Wireless Body Area Network). La antena propuesta consiste en ranuras en forma de $L$ que se han determinado en función de las bandas de frecuencia. Para la integración de estas técnicas se consideraron tres bandas de frecuencia que consisten en la banda ISM de $2,4 \mathrm{GHz}$ y $5,7 \mathrm{GHz}$, y la UWB de la banda de 3,5 $\mathrm{GHz}$, respectivamente [44].

En conclusión, la antena propuesta está diseñada con el propósito de obtener modelos SAR bajos del cuerpo humano, considerando tanto el efecto electromagnético como el modelo de caparazón en los tejidos humanos.

\subsection{Antenas planares para tags RFID pasivos en bandas UHF sobre sustrato polimérico con características de flexibilidad $y$ transparencia para la aplicación en sistema de transporte inteligente}

Los sistemas de identificación por radio frecuencia o RFID por sus siglas en inglés (Radio Frequency IDentification) son los más comunes en la actualidad para el manejo e identificación de diferentes personas y objetos, lo cual, ha permitido el desarrollo de muchas investigaciones para lograr una mejora en tecnología y aplicaciones [45].

Básicamente, el sistema centra su atención a la comunicación inalámbrica (Wireless) que hay entre una antena lectora (reader) y el tag RFID, este último, permite al dispositivo que presenta un mayor movimiento en desarrollos investigativos en cuanto a métodos de fabricación, características de funcionamiento, tamaño, flexibilidad, durabilidad, entre otras.

El tag RFID, se define como un dispositivo que se estructura básicamente por tres componentes, que son: el microchip, la antena y el sustrato [46]. 
El microchip o circuito integrado, es un dispositivo diseñado y fabricado para diversos medios y propósitos, que presentan tecnología CMOS o EEPROM. Su característica principal se fundamenta en la verificación de la información y almacenamiento temporal o permanente de información relevante para la identificación dentro del sistema [47].

El sustrato, es el material que se utiliza como una base para la fabricación del tag, el cual tiene a la antena y al microchip [48]. Este componente, es la base principal del funcionamiento de la antena, ya que permite la formación de campos electromagnéticos alrededor de él (polarización del material) y desarrolla en la irradiación de la antena.

Considerando que la antena representa el elemento central de funcionamiento del tag RFID, esta se encargará principalmente de recibir y transmitir la información en forma de campos electromagnéticos proveniente del reader 0 el microchip, respectivamente. También puede cumplir el papel de fuente de alimentación para tags RFID pasivos, transformando las señales captadas en corriente eléctrica que pueda ser aprovechada por el microchip.

En resumen, su atención a la comunicación inalámbrica (wireless) es su objetivo principal. Por otro lado, este dispositivo se caracteriza por ser de reducido tamaño debido a la medición que presenta el tag, las antenas diseñadas deben catalogarse como eléctricamente pequeñas.

\subsection{Antena plana para aplicaciones en las bandas L1 y L2 de GPS}

Desarrollo de una antena con polarización circular y doble resonancia, usando sustratos de baja constante dieléctrica y bajo precio, resultados comparables frente a diseños convencionales [49].

Estos diseños, constan de dos parches rectangulares separados por un espacio de aire, que incluyen dos cortes triangulares y dos parches parásitos ubicados en caras opuestas, logrando brindar un grado de libertad adicional que permite obtener una apropiada relación axial y acoplamiento aceptable a las frecuencias deseadas. La antena se optimizó mediante análisis paramétrico llevado a cabo sobre diversas variables que hacen parte de la geometría. El prototipo implementado permite la operación simultánea de la antena en las bandas de GPS de $1,227 \mathrm{GHz}$ (banda L2) y $1,575 \mathrm{GHz}$ (banda L1).

En relación con lo anterior, la antena presenta un ancho de banda de impedancia medido de $18,03 \mathrm{MHz}(\mathrm{fc} 1=1,227 \mathrm{GHz})$ y $17,66 \mathrm{MHz}$ (fc2 = $1,575 \mathrm{GHz}$ ), para VSWR $\leq 2: 1$, los resultados de las simulaciones para la ganancia fueron de $5,4 \mathrm{dBi}$ y $6,8 \mathrm{dBi}$ y relación axial de $8,1 \mathrm{MHz}$ y $10,2 \mathrm{MHz}$ en las bandas L2 y L1 respectivamente. Las simulaciones numéricas concuerdan con las medidas sobre el prototipo experimental probando que la antena se comporta de acuerdo con lo esperado.

\subsection{Estructura EBG para disminución de la radiación en parches}

En la sociedad actual, cada vez es más habitual la aparición de dispositivos cuya cercanía con el cuerpo humano es más significativa. Esta proximidad provoca que la disminución de la radiación emitida hacia la persona que lo porta sea de vital importancia, y es por esto, por lo que en este estudio se presentará una solución para poder mitigar en la medida de lo posible la radiación trasera [50].

Por otro lado, se estudia el comportamiento de una estructura periódica de forma aislada e integrada con un parche para evaluar el efecto que produce sobre este último. La banda de frecuencia elegida es la ISM (2.4 a $2.5 \mathrm{GHz}$ ) en la que se encuentran las comunicaciones WLAN y WPAN. El diseño se realizará en fibra de vidrio y se ampliará el estudio a las antenas textiles. Este tipo de antenas tiene como característica que están realizadas con materiales textiles y, de esta forma, pueden ir adheridas a la ropa formando parte de la vestimenta. 
Antes bien, se realiza el modelado de la estructura EBG en dos versiones diferentes, mediante vías y paredes metálicas, y en los dos substratos especificados. Se optimiza ésta para que trabaje en la frecuencia adecuada mediante diferentes estrategias. En una de ellas se hallan los diagramas de dispersión, para lo que se ha de suponer una estructura infinita, mientras que el otro método realiza simulaciones de onda completa, simulando la transmisión en dos configuraciones, con línea microstrip suspendida y sin línea.

De esta forma, se diseña el parche a la misma frecuencia y en los dos substratos elegidos, y una vez optimizada, se procede a combinar ambas estructuras. Tras la integración de la EBG se estudia de qué forma modifica el comportamiento de la antena, para ello se analiza el parámetro de reflexión, la ganancia, y mediante sondas de campo se cuantifica si se reduce la propagación de ondas de superficie [51].

También, gracias a las simulaciones realizadas, se ha comprobado como la estructura periódica ayuda a reducir la radiación trasera y a aumentar la ganancia respecto del caso en el que únicamente se disponía del parche.

Finalmente, para confirmar el funcionamiento real del diseño, se ha construido un prototipo de antena sobre fibra de vidrio que incorpora una de las configuraciones estudiadas. Comparando los resultados experimentales y los simulados, se ha comprobado como el funcionamiento es bastante similar en adaptación.

En conclusión, este tipo de antenas también tiene la característica de que está realizadas con materiales textiles y, de esta forma, pueden ir adheridas a la ropa formando parte de la vestimenta. Se diseña en fibra de vidrio como aporte al estudio de antenas textiles, este elemento se crea con el objeto de mitigar la radiación en parches y su impacto en el cuerpo humano.

\subsubsection{Rendimiento en el cuerpo humano}

La antena también se caracteriza por su influencia en el cuerpo. Se escogieron tres tipos diferentes de fantasmas humanos en la simulación para imitar el pecho, el hombro y el brazo.

Aplicando el cofre como un modelo cúbico de tres capas, la primera que tenga una capa de piel de 3 $\mathrm{mm}$ de espesor, le segunda una capa de grasa de 7 $\mathrm{mm}$ de grosor y la tercera una capa muscular de 60 $\mathrm{mm}$ de espesor. El tamaño total del fantasma es 500 $\times 500 \times 70 \mathrm{~mm} 3$. El modelo de hombro posee estructura igual en capas que el modelo de cofre, pero su tamaño es de $200 \times 100 \times 70 \mathrm{~mm} 3$. El modelo de brazo es un cilíndrico de cuatro capas, que tiene una capa de piel de $2 \mathrm{~mm}$ de grosor, una capa de grasa de $5 \mathrm{~mm}$ de espesor, una capa de músculo de $20 \mathrm{~mm}$ de grosor y un núcleo de hueso con $13 \mathrm{~mm}$. Con una longitud total del modelo cilíndrico de $350 \mathrm{~mm}$ [52].

Es decir, teniendo la antena a $10 \mathrm{~mm}$ por encima de la superficie de la piel, para imitar la separación por capas de ropa. Para el modelo de brazo, la antena se doblada en dos direcciones. Los extremos simulados y medidos son los coeficientes de fluencia. La conclusión más notable es que la banda superior (la banda de $2,4 \mathrm{GHz}$ ) casi no está influenciada por el cuerpo humano, incluso en las condiciones de flexión [53].

Por ende, la radiación disminuye casi a un $10 \%$ ya que es absorbida por el cuerpo humano. En la banda inferior, el patrón de la antena cambia de un costado a uno omnidireccional. La eficiencia de la radiación cae bruscamente sobre el cuerpo humano.

\section{CONCLUSIÓN}

Para concluir, en el presente artículo se realizó una revisión general y explicativa sobre la implementación de los metamateriales para el diseño de antenas textiles, con el principal objetivo de mostrar qué son los metamateriales y sus aplicaciones. Mostrando que los metamateriales tienen un amplio rendimiento y han traído una factible utilización en grandes aplicaciones electromagnéticas, desde los microondas hasta el 
óptico, especialmente para los dispositivos de ondas radiadas. Además, ha sido posible reducir las dimensiones de los dispositivos, mejorado su consumo energético, abarcando en el desarrollo de antenas flexibles que puedan integrarse en la ropa.

Adicionalmente se ha demostrado los grandes avances en ciertas áreas de la ingeniería y las ciencias gracias a la implementación de estas.

\section{REFERENCIAS BIBLIOGRÁFICAS}

[1] S. R. M. Beyer, Antenas Textiles, Valparaiso: Pontificia universidad catolica de valparaiso, 2014.

[2] S. Yan, V. Volskiy y Member, «Compact DualBand Textile PIFA for 433-MHz/2.4-GHz ISM Bands,» 2017. [En línea].

[3] F. Pizarro, A. Leiva y M. Rodrıguez, «DualBand Textile Patch Antenna for GSM-WiFi Bands Using EVA-Foam as Substrate," Pontificia Universidad Catolica de Valparaıso, Chile, 2016.

[4] B. Babusiaka, S. Borika y L. Balogovab, "Textile electrodes in capacitive signal sensing applications,» 2017.

[5] J. Virkki, Z. Wei, A. Liu, L. Ukkonen y T. Björninen, «Wearable Passive E-Textile UHF RFID Tag Based on a Slotted Patch Antenna with Sewn Ground and Microchip Interconnections,» Ming-ChunTang, 2017.

[6] D. Ferreira y P. Pires, «Wearable textile Antennas,» Digital Object Identifier, 2017.

[7] E. Asensio, «Estructura EBG para disminución de la radiación en parches,» Universidad Carlos III de Madrid, Leganes, 2012.

[8] R. M. F. M. J. \&. M. F. Marques, «Comparative analysis of edge- and broadside- coupled split ring resonators for metamaterial design - theory and experiments,» IEEE Trans. Ant. Propagat, pp. 2572-2581, 2003.

[9] Ministerio de la defensa, "Los metamateriales y sus aplicaciones en defensa,» Monografías del SOPT, pp. 1-142, 2011.

[10] W. Hidalgo, «Diseño de antenas planares para tags RFID pasivos en bandas UHF sobre sustrato polimérico con características de flexibilidad y transparencia para la aplicación en sistema de transporte inteligente,» Universidad Nacional de Colombia , Bogota, 2017.

[11] L. \&. L. J. L. Wang, «A Novel Metamaterial Microstrip Antenna of Broadband and HighGain,» Proceedings of ISAP2012, pp. 806-809, 2006.

[12] B.-I. W. W. P. J. C. X. G. T. M. \&. K. J. A. Wu, "A study of using metamaterials as antenna substrate to enhance gain,» Progress In Electromagnetics Research., pp. 295-328., 2005.

[13] J. S. J. P. S. D. E. \&. Y.-K. Y. Xiaoyu Cheng, «Reconfigurable split ring resonator array loaded waveguide for insitu tuning,» IEEE International Symposium on Antennas and Propagation (APSURSI), pp. 2947-2950, 2011.

[14] R. Zhou y H. \&. Z. H. Xin, «Liquid-based dielectric resonator antenna and its application for measuring liquid real permittivities," Microwaves, Antennas Propagation, p. 255262, 2014.

[15] R. Salvado, C. Loss, R. Gonçalves y P. Pinho, «Textile Materials for the Design of Wearable Antennas,» Sensors, 2012.

[16] M. Stoppa y A. Chiolerio, «Wearable Electronics and Smart Textiles: A Critical Review,» Sensors, 2014. 
[17] H. Kaschel, «Design of a tri-band antenna microstrip for a WBAN the low SAR,» Universidad de Santiago de Chile, 2016.

[18] J. Santiso, «DISEÑO DE UNA ANTENA MULTIMODO SOBRE SUBSTRATO TEXTIL PARA APLICACIONES CORPORALES,» Universidad Politecnica de Valencia, Valencia, 2010.

[19] L. Castellanos y F. López, «Metamateriales: principales características y aplicaciones,» Rev. Acad. Colombia, 2016.

[20] R. O. R. E. J. D. A. R. F. K. \&. T. A. Ouedraogo, «Miniaturization of Patch Antennas Using a Metamaterial-Inspired Technique,» IEEE Transactions on Antennas and Propagation, pp. 2175-2182., 2012.

[21] F. M. a. R. R.-E.-I. R. Marqués, ««Role of bianisotropy in negative permeability and lefthanded metamaterials, ", " Physical Review B, vol. 6, pp. 144-440, 2002.

[22] J. M. F. M. a. F. M. R. Marqués, «Left-handedmedia simulation and transmission of $\mathrm{EM}$ waves in sub-wavelength split-ring resonator loaded metallic waveguides,» Physical Review Letters, pp. 18-28, 2002.

[23] Q. W. B.-S. J. W. H.-L. \&. J. Fan-Yi Meng, "Comments on "Waveguide Miniaturization Using Uniaxial Negative Permeability Metamaterial,» IEEE Transactions on Antennas and Propagation, p. 55, 2007.

[24] Z. A. L. V. \&. N. E. Jacob, "Optical Hyperlens: Far-field imaging beyond the diffraction limit., Optics Express, p. 14, 2006.

[25] S. C. K. L. \&. A. P. Chaimool, «Simultaneous gain and bandwidths enhancement of a singlefeed circularly polarized microstrip patch antenna using a metamaterial reflective surface,» Progress In Electromagnetics Research B, pp. 23-37, 2010.

[26] Y.-A. C. I.-L. \&. C. L.-W. Chen, «Spiral hyperlens with enhancements of image resolution and magnification,» Journal of Modern Optics, pp. 1029-1034, 2016.

[27] X. S. D. E. K. C. \&. Y. Y.-K. Cheng, «A Compact Omnidirectional Self-Packaged Patch Antenna With Complementary Split-Ring Resonator Loading for Wireless Endoscope Applications.," IEEE Antennas and Wireless Propagation Letters, pp. 10: 1532-1535, 2011.

[28] M. C. D. \&. R. E. Domínguez, «Design a sensor of relative dielectric permittivity of a medium using an antenna microstrip with metamaterial structures,» Actas de Ingeniería, pp. 110-114, 2015.

[29] C. \&. I. T. Caloz, «Electromagnetic Metamaterials: Transmission Line Theory and Microwave Applications.,» I. John Wiley \& Sons, Ed.) (First.). Hoboken, NJ, USA: John Wiley \& Sons, Inc., 2005.

[30] D. S. D. E. L. F. \&. R.-V. E. Catano-ochoa, «erformance Analysis of a Microstrip Patch Antenna loaded with an Array of Metamaterial Resonators.," IEEE International Symposium on Antennas and Propagation/ USNC-URSI National Radio Science, pp. 1-3, 2016.

[31] A. E.Úbeda, ««Radiation pattero of composite finite arrays of conductingelements with anexcitingelementary dipole», , IEEE Ante/mas Propagat. Sociery Int. Symp, pp. 379-382, 2003.

[32] A. W. W. A.-S. S. \&. A. D. Ebrahimi, «HighSensitivity Metamaterial-Inspired Sensor for Microfluidic Dielectric Characterization.,» IEEEA Sensors Journal., p. 14, 2014. 
[33] J. B. F. F. M. S. a. R. M. F. Martin, ««Split ring resonator-based left-handed coplanar waveguide, , , Applied Physicssics Letters, vol. 83, p. 4652-4654, 1 Diciembre 2003.

[34] F. L. T. L. M. A. G. B. J. D. B. J. B. M. S. M. Falcone, «Babinet Principle Applied to the Design of Metasurfaces and Metamaterials,» Physical Review Letters, p. 93, 2004.

[35] D. C. O. E. R. V. María Domínguez V., «Design a sensor of relative dielectric permittivity of a medium using an antenna microstrip with metamaterial structures, " Actas de Ingeniería, vol. 1, pp. 110-114, 2015.

[36] D. W. W. S.M.Rao, «Electromagnetic scattering by surfaces of arbitrary shape,» IEEE Trans Antennas Propagar, pp. 409-418, 1982.

[37] D. P. W. V. D. N.-N. S. \&. S. Smith, «Composite Medium with Simultaneously Negative Permeability and Permittivity," Physical Review Letters., vol. 84, pp. 4184-4187, 2000.

[38] H. T. Chen, W. Padilla, J. Zide y A. Gossard, "Active terahertz metamaterial devices," Nature, p. 597-600, 2006.

[39] Y. Dong, «Metamaterial-Based Antennas,» IEEE, pp. 2271 - 2285, 2012.

[40] T. \&. R. O. M. Almoneef, «Split-ring resonator arrays for electromagnetic energy harvesting.," Progress In Electromagnetics Research B, 62 (January), pp. 167-180, 2015.

[41] J. D. B. J. M. F. S. R. M. F. F. L. T. S. M. Baena, «Equivalent-circuit models for split-ring resonators and complementary split ring resonators coupled to planar transmission lines,» IEEE Transactions on Microwave Theory and Techniques, p. 53, 2005.
[42] M. Bogosanovich, «Microstrip patch sensor for measurement of the permittivity of homogeneous dielectric materials,» IEEE Transaction Instrument Measurement, p. 1144-1148., 2000.

[43] O. Y. T. \&. O. J. Büyüköztürk, «A methodology for determining complex permittivity of construction materials based on transmissiononly coherent, wide-bandwidth free-space measurements," Cement and Concrete Composites, p. 349-359, 2006.

[44] M. \&. A. M. Tauseef Asim, "Metamaterial Inspired Microstrip Antenna Investigations Using Metascreens,» International Journal of Antennas and Propagation, pp. 1-9, 2015.

[45] J. Lesnikowski, «Dielectric Permittivity measurement methods of textile substrate of textile transmission lines,» Przeglad Elektrotechniczny, p. 148-151, 2012.

[46] L.-W. L. Y.-N. S. Y. T. M. J. R. \&. M. O. J. F. Li, "Addendum: A broadband and high-gain metamaterial microstrip antenna,» Applied Physics Letters, 2011.

[47] S. Linden, «Magnetic Response of Metamaterials at 100 Terahertz,» Science, pp. 1351-1353., 2004.

[48] S. E. C. D. G. K. M. W. Z. J. K. T. W. M. Linden, «Photonic Metamaterials Magnetism at Optical Frequencies,» IEEE Journal of Selected Topics in Quantum Electronics, vol. 12, pp. 10971105.

[49] V. Veselago, «The Electrodynamics of substances with simultaneously negative values of $\varepsilon$ and $\mu$.," Soviet Physics Uspekhi, pp. 509-514, 1968.

[50] G. E. J. S. K. H. D. L. J. S. P. U. Y. Lipworth, «Magnetic metamaterial superlens for 
increased range wireless power transfer," Scientific Reports, p. 4: 3642, 2014.

[51] F. L. E. R.-V. Luis M. Castellanos, «Metamateriales: principales características y aplicaciones,» Facultad de Ingenierías, Instituto Tecnológico Metropolitano, Medellín, Colombia, 2016.

[52] C. C. C. a. T. I. C. Caloz, ««Full-wave verification of the fundamental properties of left-handed materials in waveguide configurations," Journal of Applied Physics, p. 5483-5486, 2001.

[53] a. R. W. Z. C. Y. Cheng, «Tailoring doublenegative metamaterial responses to achieve anomalous propagation effects along microstrip transmission lines,» IEEE Trans. Microwave Theory \& Tech., pp. 2306-2314, 2003.

[54] C. Suárez, J. López y G. P. Leguizamón, «Antena plana para aplicaciones en las bandas L1 y L2 de GPS, Revista Científica Ingeniería y Desarrollo, 2016.

[55] E. Ú. y. J. R. J. M González-Arbesú, «METAMATERIALES EN MICROONDAS $Y$ ANTENAS,» Universitat Politecnica de Catalunya, Campus, pp. 32-38, Diciembre 2003.

[56] B. \&. L. B. Kapilevich, «Application of resonant monopole antenna for determination of complex permittivity of liquids, powdered and granular materials,» Measurement, p. 19641969, 2013. 
